\title{
The Role of CD10 Staining in Distinguishing Invasive Endometrial Adenocarcinoma from Adenocarcinoma Involving Adenomyosis
}

\author{
Alessandra F. Nascimento, M.D., Michelle S. Hirsch, M.D., Ph.D., Aida Cviko, M.D., Ph.D., \\ Bradley J. Quade, M.D., Ph.D., Marisa R. Nucci, M.D. \\ Department of Pathology, Division of Women's and Perinatal Pathology, Brigham and Women's Hospital \\ and Harvard Medical School, Boston, Massachusetts
}

\begin{abstract}
Adenomyosis may be involved by endometrial adenocarcinoma, but in contrast to true myometrial invasion, the depth of an adenomyotic focus involved by carcinoma does not alter pathologic tumor staging. Therefore, distinction from carcinoma invading myometrium is clinically relevant. We hypothesized that CD10, a marker of non-neoplastic and neoplastic endometrial stroma, would highlight the stromal component of adenomyotic foci and be useful in this distinction. Thirty-nine cases of endometrial adenocarcinoma were analyzed and divided into three groups: $I$, invasive endometrial adenocarcinoma ( $n=14)$; II, endometrial adenocarcinoma involving adenomyosis but without myometrial invasion ( $n=18$ ); and III, adenomyosis involved by endometrial adenocarcinoma with concomitant invasive component $(n=7)$. All cases of adenomyosis involved by endometrial adenocarcinoma demonstrated CD10 expression in the stromal cells of adenomyotic foci. Eleven of 21 cases (52\%) of invasive adenocarcinoma also showed CD10 expression, at least focally, in cells immediately surrounding the infiltrating glands. Of these, two cases (from Group III) also had associated adenomyotic involvement by carcinoma. The remaining cases of invasive carcinoma were negative for $\mathrm{CD10}$. Therefore, presence of CD10 staining immediately surrounding neoplastic glands does not equate with involvement of adenomyosis by endometrial adenocarcinoma.
\end{abstract}

\footnotetext{
Copyright (C) 2003 by The United States and Canadian Academy of Pathology, Inc.

VOL. 16, NO. 1, P. 22, 2003 Printed in the U.S.A.

Date of acceptance: October 3, 2002.

Supported by a grant (RPG CNE-100283) from the American Cancer Society (BJQ).

Presented in part at the United States and Canadian Pathology Meeting, Chicago, IL, 2002.

Address reprint requests to: Marisa R. Nucci, M.D., Department of Pathology, Division of Women's and Perinatal Pathology, Brigham and Women's Hospital, 75 Francis Street, Boston, MA 02115; e-mail: mnucci@partners.org; fax: 617-277-9015.

DOI: 10.1097/01.MP.0000043523.03519.FC
}

In contrast, absence of $\mathrm{CD} 10$ expression excludes involvement of adenomyosis by adenocarcinoma.

KEY WORDS: Adenomyosis, CD10, Endometrial adenocarcinoma, Endometrial stroma.

Mod Pathol 2003;16(1):22-27

Adenomyosis is usually defined as endometrial glands and stroma located in the wall of the uterus at least one low-power field away from the surface endometrium. It affects reproductive-aged women with a history of multiple pregnancies, appears to be associated with estrogen therapy $(1,2)$, and may even be a clonal process (3). Although endometrial adenocarcinoma may arise in foci of adenomyosis (4-9), adenomyosis is more often secondarily involved by endometrial adenocarcinoma that has arisen in surface endometrium. The distinction between this condition and true myometrial invasion by endometrial adenocarcinoma may sometimes be difficult, particularly if there is extensive involvement of adenomyosis by adenocarcinoma, if the secondarily involved adenomyosis extends deeply into the uterine wall, or if the presence of adenomyosis is masked by subtotal replacement by adenocarcinoma. This distinction is important because pathological staging, and consequentially, therapy is dependent on the presence and depth of invasion (10). In contrast, involvement of adenomyosis by endometrial adenocarcinoma carries no adverse prognostic significance $(11,12)$. We hypothesized that CD10, a marker of non-neoplastic and neoplastic endometrial stroma (13-15), would be useful in this distinction by highlighting the stroma of adenomyotic foci involved by endometrial adenocarcinoma and being absent around foci of invasion.

\section{MATERIALS AND METHODS}

Thirty-nine cases corresponding to hysterectomy specimens removed between the years of 1998 and 
2001 for pathological diagnoses of endometrial adenocarcinoma were retrieved from the surgical files of the Women's and Perinatal Pathology Division of the Brigham and Women's Hospital (Boston, MA). In all cases, hematoxylin-and-eosin (H\&E) stained sections were available for review, and the diagnoses were confirmed. The endometrial carcinoma was graded using the FIGO staging system (10).

The cases were subsequently divided into three groups. Group I consisted of cases of invasive endometrial adenocarcinoma. Group II included specimens with endometrial adenocarcinoma involving adenomyosis but without myometrial invasion. Group III was composed of cases with adenomyosis involved by endometrial adenocarcinoma with coexistent myometrial invasion. Invasion of the myometrium was based on established criteria (16); features of invasion included irregular pattern of infiltration, irregular glandular outlines, alterations in the cytomorphology of the neoplastic cells (particularly eosinophilic change to the cytoplasm), and presence of periglandular desmoplasia, edema, and inflammation.

All cases were stained for CD10 (Novocastra Laboratories, LTD, Tyne, U.K.) using mouse monoclonal antibody (clone 56C6), at a dilution of 1:80. After high-temperature antigen retrieval, the sections were incubated for 60 minutes with the primary antibody at $25^{\circ} \mathrm{C}$, followed by the standard $\mathrm{ABC}$ technique. The stain was considered positive when present, at least focally, immediately surrounding the neoplastic glands. Non-neoplastic surface endometrial stromal cells and stroma present in uninvolved foci of adenomyosis were used as an internal positive control.

Nine cases in which there was available material (seven cases in Group I and two cases in Group III) were stained with antibody to the muscle markers desmin (monoclonal clone D33; dilution 1:500; DAKO Corporation, Carpinteria, CA) and h-caldesmon (monoclonal clone h-CD; dilution 1:300; DAKO). The latter antibody required 30 minutes of antigen retrieval. The myometrium was used as an internal positive control.

\section{RESULTS}

A summary of the immunohistochemical results for CD10 in all groups is summarized in Table 1.

\section{Group I: Invasive Endometrial Adenocarcinoma}

Group I included 14 cases of invasive endometrial adenocarcinoma. Patients' ages ranged between 49 and 77 years (median, $60.5 \mathrm{y}$ ). Twelve of 14 cases $(85.7 \%)$ were diagnosed as endometrial adenocarcinoma, endometrioid subtype. Two cases had mixed endometrioid, papillary serous, and clear cell features. Six cases were Grade 1, four were Grade 2, and four were Grade 3 (of 3 grades). Three of fourteen cases $(21.4 \%)$ showed additional uninvolved foci of adenomyosis. All tumors but one showed myometrial invasion ranging from 10 to $95 \%$ of the myometrial thickness (median 50\%); one adenocarcinoma superficially invaded a submucosal leiomyoma.

Five of the 14 cases (35.8\%) showed absence of CD10 staining in cells adjacent to the invasive glands (Fig. 1A-B). However, 9 of the 14 cases (64.2\%) revealed CD10 expression in cells immediately surrounding the neoplastic glands (Fig. 2A-B). Of these nine cases, four cases had a circumferential staining pattern, whereas five showed only focal staining $(<50 \%$ of the gland circumference). In eight of the nine cases (89\%) with CD10 expression surrounding invasive glands, CD10-expressing cells were part of a desmoplastic reaction. In the remaining case, there was no desmoplastic reaction by $H \& E$ examination and, although the invasive border was rounded, the extent and pattern of invasion were consistent with invasive adenocarcinoma.

Immunoperoxidase stains for desmin and h-caldesmon, performed in seven of the nine tumors with CD10-expressing cells, revealed in five of them scattered rare positive cells for both markers in the cells that were also positive for CD10, including the one tumor with a rounded border of invasion. The staining was weaker than in the adjacent myometrium. In the other two cases in which CD10 was positive, both muscle markers were negative in the cells immediately surrounding the invasive

TABLE 1. Summary of CD10 Staining

\begin{tabular}{cccc}
\hline Group & $\begin{array}{c}\text { CD10+ } \\
\text { Invasive }\end{array}$ & $\begin{array}{c}\text { CD10- } \\
\text { Invasive }\end{array}$ & $\begin{array}{c}\text { CD10+ } \\
\text { Adenomyosis }\end{array}$ \\
\hline I & $9 / 14^{a}(64.2)$ & $5 / 14(35.7)$ & N/A \\
II & N/A & N/A $/ A ~$ & $18 / 18(100)$ \\
III & $2 / 7^{b}(28.5)$ & $5 / 7(71.4)$ & $0 / 18(0)$ \\
$0 / 7(0)$ & $(100)$ \\
\hline
\end{tabular}

All data are $n(\%)$.

N/A, not applicable; Group I, invasive endometrial adenocarcinoma; Group II, endometrial adenocarcinoma involving adenomyosis without myometrial invasion; Group III, endometrial adenocarcinoma involving adenomyosis with myometrial invasion.

${ }^{a}$ Focal in five cases.

${ }^{b}$ Focal in one case. 

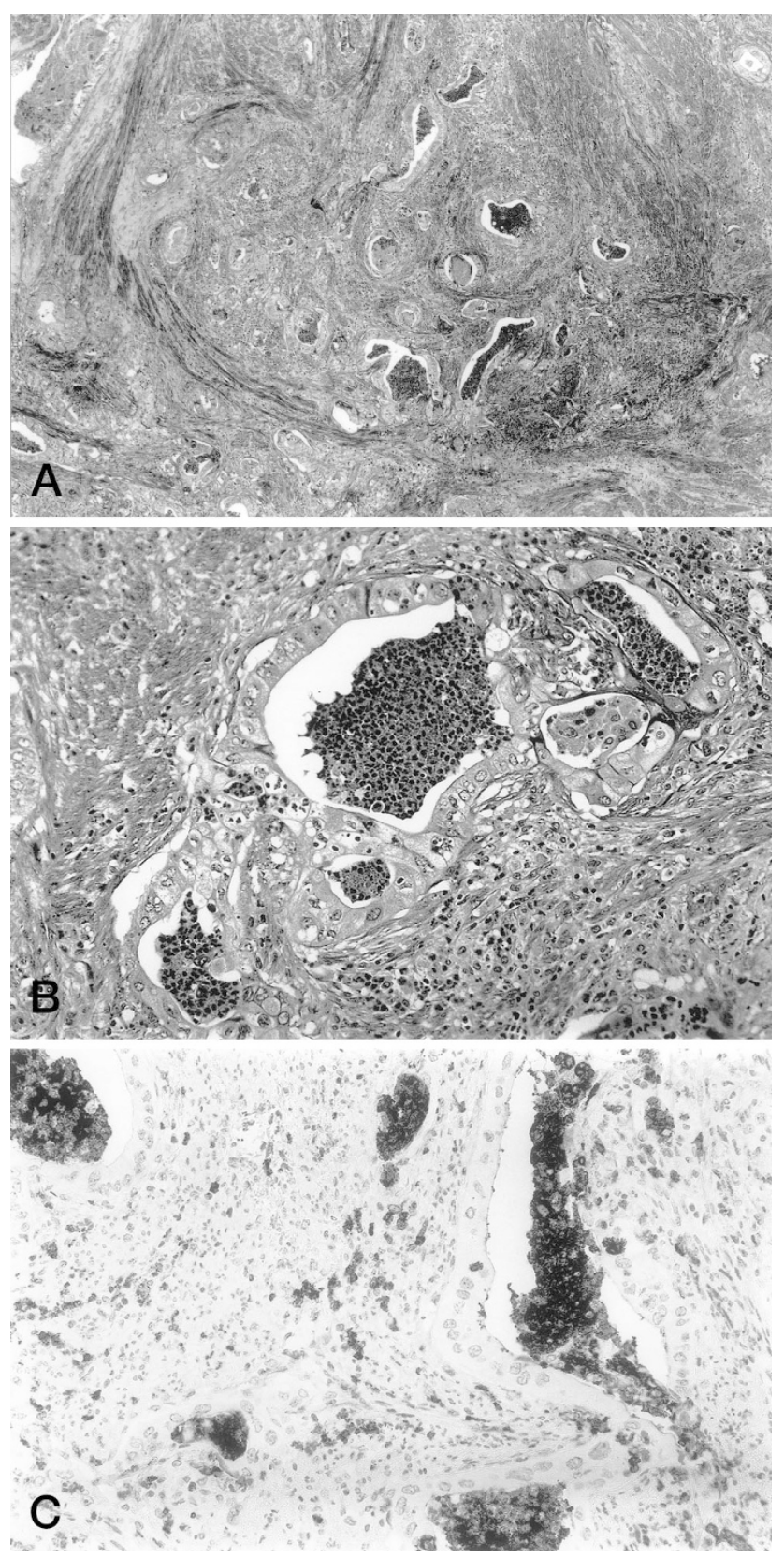

FIGURE 1. A, B, invasive endometrial adenocarcinoma (H\&E stain). C, lack of CD10 staining around the neoplastic glands. Intraluminal and stromal granulocytes are an internal positive control.

glands. In general, the expression of the muscle markers was weaker than in the surrounding myometrium and more focal than in the CD10expressing cells (Fig. 3A-B).

Group II: Endometrial Adenocarcinoma Involving Adenomyosis But without Myometrial Invasion

Group II included 18 cases of endometrial adenocarcinoma involving adenomyosis without myometrial invasion. Patients' ages ranged from 40 to 83 years of age (median, 60.5 y). All carcinomas were of the endometrioid subtype, with a predominance of Grade 1 tumors (13 of 18; 72.2\%). Four

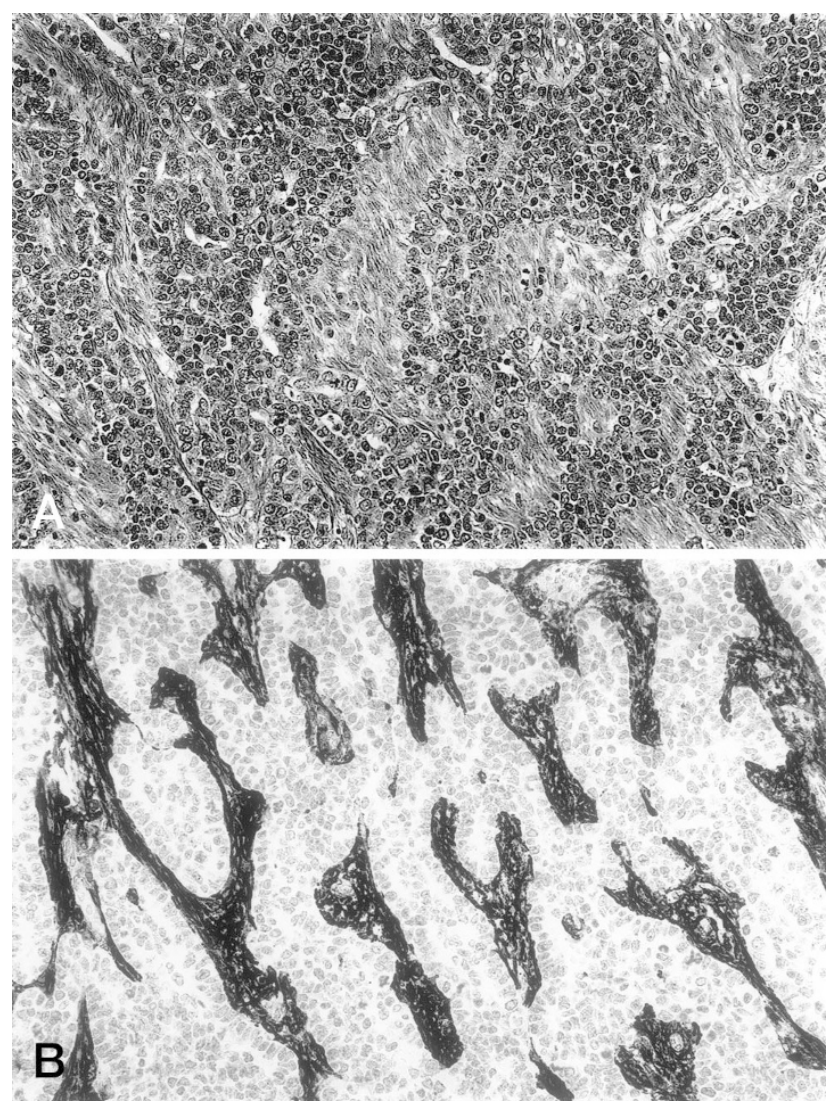

FIGURE 2. A, invasive adenocarcinoma with accompanying

desmoplastic reaction (H\&E stain). B, CD10-expressing cells are present immediately adjacent to the invasive focus.

tumors were classified as Grade 2 and one as Grade 3 (of 3). Two cases also had foci of adenomyosis uninvolved by carcinoma. Strong and diffuse CD10 expression in the endometrial stromal cells of adenomyotic foci immediately around neoplastic glands was present in all cases (Fig. 4A-B).

Group III: Adenomyosis Involved by Endometrial Adenocarcinoma with Coexistent Myometrial Invasion

Group III included seven cases of endometrial adenocarcinoma with involvement of adenomyosis and coexistent myometrial invasion. Patient age ranged between 36 and 69 years (median 55 y). Six of seven cases $(85.7 \%)$ were subclassified as endometrioid type; one case had an admixed papillary serous component, which accounted for approximately $20 \%$ of the tumor. The depth of invasion varied from 25 to $60 \%$ of the myometrial thickness (median 40\%). Four of seven tumors (57.1\%) were Grade 2, one tumor was Grade 1, and two tumors were Grade 3 (of 3 grades).

All tumors showed CD10 staining around foci of adenomyosis (Fig. 5); however, two cases (28.5\%) also exhibited CD10 staining immediately surrounding foci of invasive adenocarcinoma. In the 


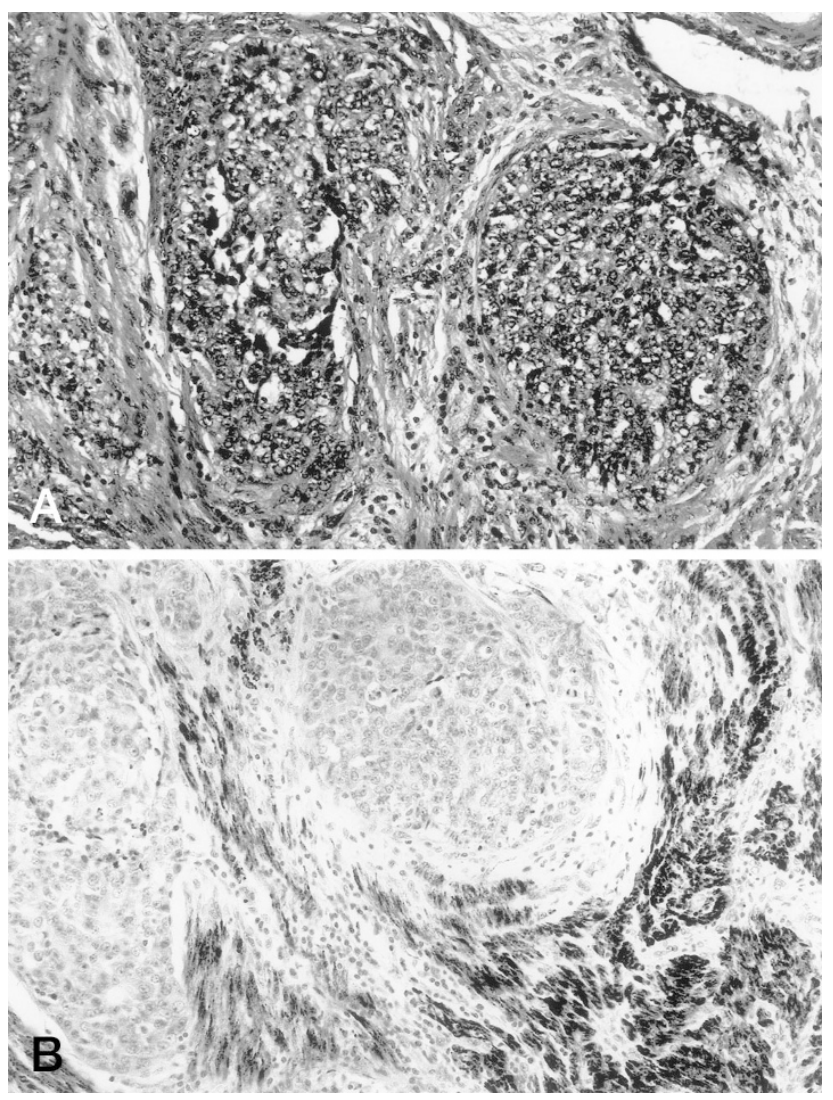

FIGURE 3. A, invasive endometrial adenocarcinoma (H\&E stain). B focal desmin-expressing cells are present immediately adjacent to the invasive glands (myometrium as internal positive control); these cells also expressed CD10.

other five cases, cells surrounding the invasive glands were negative for CD10 (Fig. 5). Correlation between $\mathrm{H} \& \mathrm{E}-$ and immunoperoxidase-stained sections demonstrated that one of the two tumors with CD10-positive cells immediately adjacent to invasive carcinoma corresponded with a peritumoral desmoplastic reaction. The other case showed direct invasion into the myometrium without definitive desmoplastic reaction. In these two cases, the muscle markers for desmin and caldesmon were also positive in these peritumoral cells, although in a weaker and more focal manner compared with surrounding myometrium.

\section{DISCUSSION}

The prevalence of adenomyosis is quite variable, ranging from 10 to $88 \%$, probably reflecting differing criteria and low diagnostic reproducibility (1720). Diagnostic criteria for adenomyosis varies widely, but the most widely accepted one is the separation of a candidate focus from the surface endometrium by at least one low-power field $(4 \times$ magnification). Recently, a distance of $\geq 3 \mathrm{~mm}$ from the endomyometrial junction and the presence of concentric myometrial hyperplasia has been pro-
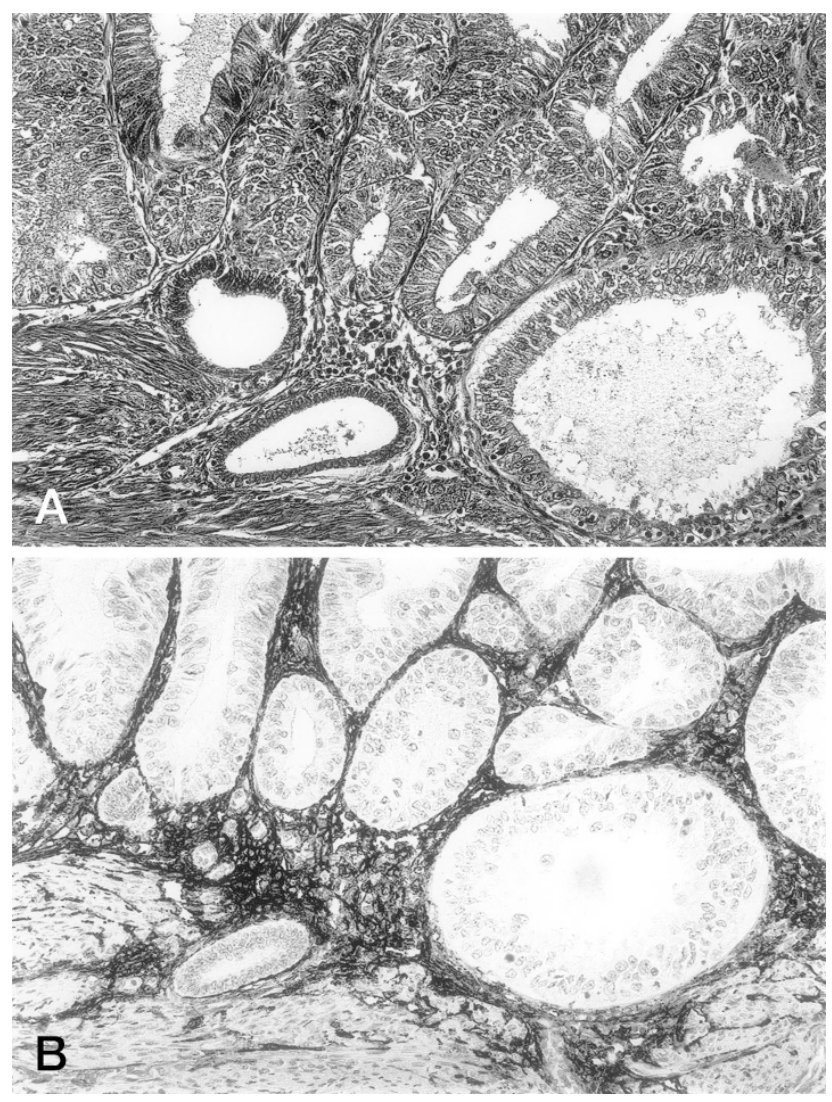

FIGURE 4. A, partial replacement of adenomyotic focus by endometrial adenocarcinoma without myometrial invasion (H\&E stain). B, the stromal cells of the adenomyotic focus are positive for CD10.

posed as more reliable diagnostic criterion (17). The risk factors for and the pathophysiology of adenomyosis are unclear. Multiparity (21) and constant estrogen exposure, such as tamoxifen therapy $(1,2)$, are believed to be associated with its development. Pandis et al. (3) found clonal deletion of the long arm of chromosome 7 in three cases, raising the possibility of a neoplastic process.

Adenomyosis may be associated with or be concurrently involved by endometrial hyperplasia and adenocarcinoma. The latter situation may, in some instances, give rise to diagnostic difficulty with regard to assessment of depth of myometrial invasion. This distinction is clinically relevant because depth of invasion is an important independent prognostic factor for patients with endometrial adenocarcinoma (10). Although adenomyosis might be located deep within the myometrium, its involvement by endometrial adenocarcinoma does not alter the patient's stage. Deep involvement of adenomyosis by endometrial adenocarcinoma without myometrial invasion has been shown to have a more favorable prognosis than tumors invasive to a similar depth $(11,12)$. In a study of 18 patients, Mittal et al. (22) showed that adenocarcinomas of low histologic grade confined to adenomyosis had an excellent prognosis. 

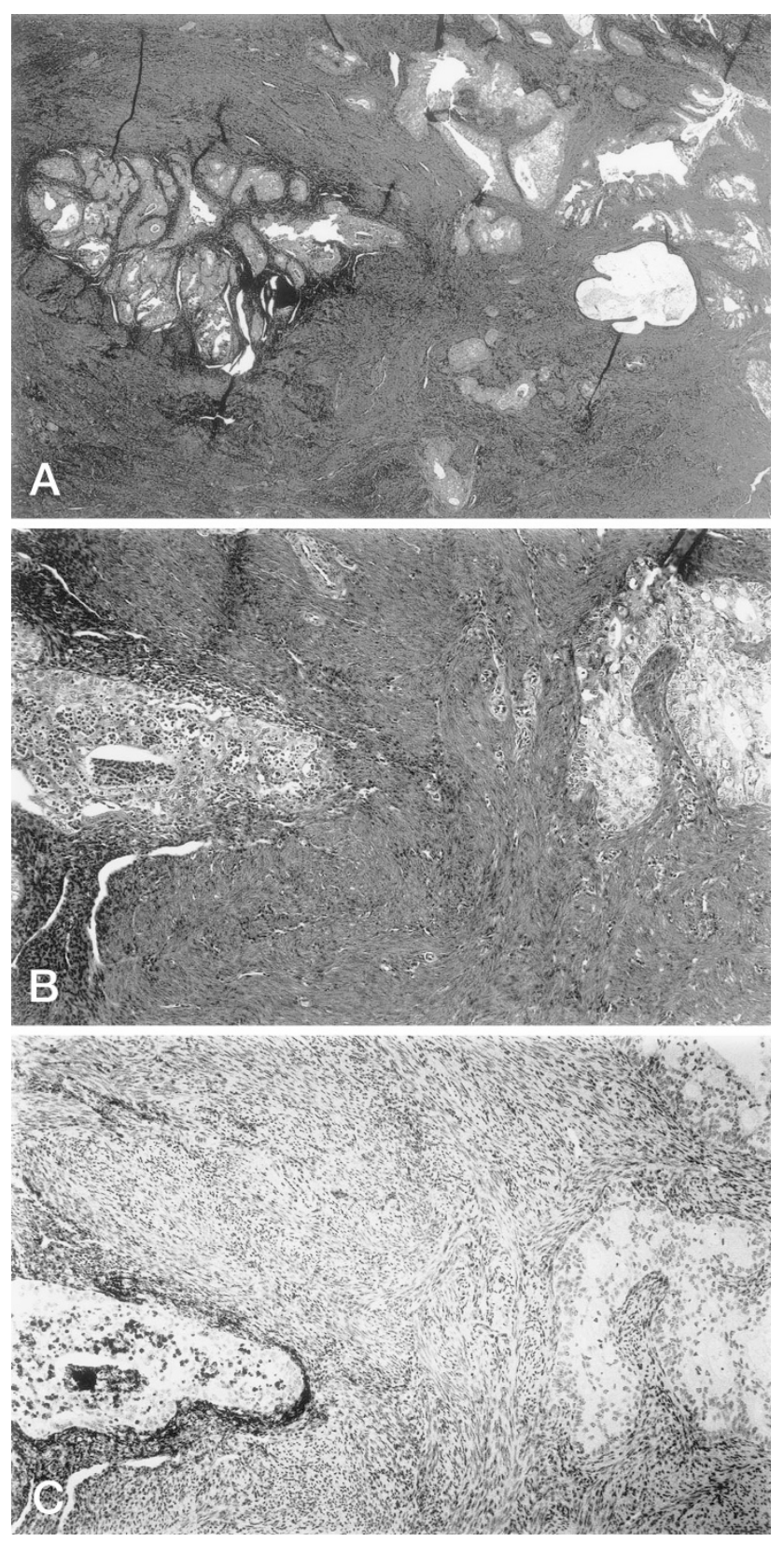

FIGURE 5. A, B, endometrial adenocarcinoma with involvement of adenomyosis and coexistent myometrial invasion. Focus of adenomyosis involved by endometrial adenocarcinoma (on $\boldsymbol{l e f t}$ ) and focus of invasive tumor (on right). C, CD10 is positive in the stromal cells in the adenomyotic focus but absent in cells surrounding the invasive focus.

Common acute lymphoblastic leukemia antigen (CALLA), or CD10, is a monomeric Type II integral membrane peptidase expressed by lymphoid progenitor cells found in normal bone marrow and thymus, and by mature granulocytes (23). It also is expressed in some acute lymphoid leukemias, such as acute lymphoblastic leukemia, and some nonHodgkin's lymphomas, such as follicular and Burkitt's lymphomas (23). Besides hematopoietic tissues and malignancies, non-neoplastic nonhematopoietic tissues such as renal tubular and glomerular cells, small intestine epithelium, normal and malignant cells of the epithelium of the stomach and colon, and myoepithelial cells of the breast also express CD10 (24-27). CD10 has also been shown to stain non-neoplastic and neoplastic endometrial stroma and thus may be helpful in the differential diagnosis of uterine stromal and smooth muscle neoplasm $(13,15,28)$. On the basis of these findings, we hypothesized that CD10 would be a useful biomarker in the distinction of adenomyosis involved by endometrial adenocarcinoma from myoinvasive endometrial adenocarcinoma. Because adenomyotic foci contain endometrial stroma, CD10 expression would be expected to be present surrounding neoplastic glands involving such foci. In contrast, CD10-positive cells would not be expected to be present around adenocarcinoma invading myometrium.

Our results demonstrate that endometrial adenocarcinoma involving adenomyosis is surrounded by CD10-expressing stromal cells reflecting the expected presence of endometrial stroma within adenomyotic foci. However, in cases of invasive adenocarcinoma, with or without associated adenomyotic involvement by carcinoma, invasive neoplastic glands were at least partially bounded by a rim of CD10-expressing cells in 11 of 21 (52\%) cases. The cells expressing CD10 corresponded to desmoplastic stromal cells in 9 cases (82\%). In the remaining 2 cases, CD10-expressing cells not associated with desmoplasia were present surrounding the invasive glands. Possible explanations for CD10 staining in these 11 cases include the following: (1) the positive cells are endometrial stromal cells and the foci interpreted as invasion are actually occult involvement of adenomyosis, rather than true myometrial invasion; (2) expression of CD10 by myometrial smooth muscle cells; (3) expression of CD10 by fibroblasts within the desmoplastic reaction; (4) spurious expression of a CD10 epitope in another protein; (5) tumor invasion accompanied by endometrial stroma; and (5) possible induction of stromal differentiation by the invasive tumor.

The first proposition, occult involvement of adenomyosis by carcinoma, can be excluded because the cases in this study were carefully examined and established criteria were cautiously applied to avoid this pitfall. Furthermore, the neoplastic glands, with or without surrounding desmoplastic reaction, showed an invasive pattern with irregular borders and absence of endometrial stroma or residual uninvolved endometrial glands indicative of adenomyosis. The separation of endometrial stroma from myometrium by $\mathrm{CD} 10$ expression is hindered by the observation that CD10 can be weakly and focally expressed by the myometrial smooth muscle (28). The application of desmin and h-caldesmon did not clearly identify the CD10-expressing cells immediately surrounding the invasive carcinoma as myometrium. Although 
desmin and caldesmon were positive in these cells, the staining pattern was weaker and more scattered when compared with the surrounding myometrium. In our opinion, these findings did not support a smooth muscle origin for these cells. Expression of CD10 by fibroblasts within the desmoplastic reaction, spurious expression of a CD10 epitope in another protein, tumor invasion accompanied by endometrial stroma, and possible induction of stromal differentiation by the invasive tumor are all reasonable possibilities that may be more definitively addressed by other, perhaps molecular, studies in the future.

In summary, involvement of adenomyosis by endometrial adenocarcinoma and its differentiation from invasive endometrial adenocarcinoma may in some instances be diagnostically difficult. CD10 expression reliably identifies adenomyosis, but CD 10 expression may also be seen around foci of invasive carcinoma. Thus, the presence of CD10 immediately surrounding neoplastic glands does not equate with involvement of adenomyosis by endometrial adenocarcinoma, whereas absence of CD10 expression is indicative of invasive endometrial adenocarcinoma and excludes involvement of adenomyosis.

\section{REFERENCES}

1. Cohen I, Beyth Y, Tepper R, Figer A, Shapira J, Cordoba M, et al. Adenomyosis in postmenopausal breast cancer patients treated with tamoxifen: a new entity? Gynecol Oncol 1995; 58:86-91.

2. Cohen I, Beyth Y, Shapira J, Tepper R, Fishman A, Cordoba M, et al. High frequency of adenomyosis in postmenopausal breast cancer patients treated with tamoxifen. Gynecol Obstet Invest 1997;44:200-5.

3. Pandis N, Karaiskos C, Bardi G, Sfikas K, Tserkezoglou A, Foliou S, et al. Chromosome analysis of uterine adenomyosis. Detection of the leiomyoma-associated $\operatorname{del}(7 \mathrm{q})$ in three cases. Cancer Genet Cytogenet 1995;80:118-20.

4. Winkelman J, Robinson R. Adenocarcinoma of endometrium involving adenomyosis. Report of an unusual case and review of the literature. Cancer 1966;19:901-8.

5. Hernandez E, Woodruff JD. Endometrial adenocarcinoma arising in adenomyosis. Am J Obstet Gynecol 1980;138:827-32.

6. Woodruff JD, Erozan YS, Genadry R. Adenocarcinoma arising in adenomyosis detected by atypical cytology. Obstet Gynecol 1986;67:145-8.

7. Takai N, Akizuki S, Nasu K, Etoh Y, Miyakawa I. Endometrioid adenocarcinoma arising from adenomyosis. Gynecol Obstet Invest 1999;48:141-4.

8. Sasaki T, Sugiyama T, Nanjo H, Hoshi N, Murakami M, Sugita A, et al. Endometrioid adenocarcinoma arising from adenomyosis: report and immunohistochemical analysis of an unusual case. Pathol Int 2001;51:308-13.

9. Koshiyama M, Suzuki A, Ozawa M, Fujita K, Sakakibara A, Kawamura M, et al. Adenocarcinomas arising from uterine adenomyosis: a report of four cases. Int J Gynecol Pathol 2002;21:239-45.

10. Benedet JL, Bender H, Jones H III, Ngan HY, Pecorelli S. FIGO staging classifications and clinical practice guidelines in the management of gynecologic cancers. FIGO Committee on Gynecologic Oncology. Int J Gynaecol Obstet 2000;70: $209-62$.
11. Hall JB, Young RH, Nelson JH Jr. The prognostic significance of adenomyosis in endometrial carcinoma. Gynecol Oncol 1984;17:32-40.

12. Jacques SM, Lawrence WD. Endometrial adenocarcinoma with variable-level myometrial involvement limited to adenomyosis: a clinicopathologic study of 23 cases. Gynecol Oncol 1990;37:401-7.

13. Chu P, Arber DA. Paraffin-section detection of CD10 in 505 nonhematopoietic neoplasms. Frequent expression in renal cell carcinoma and endometrial stromal sarcoma. Am J Clin Pathol 2000;113:374-82.

14. Chu PG, Arber DA, Weiss LM, Chang KL. Utility of CD10 in distinguishing between endometrial stromal sarcoma and uterine smooth muscle tumors: an immunohistochemical comparison of 34 cases. Mod Pathol 2001;14:465-71.

15. McCluggage WG, Sumathi VP, Maxwell P. CD10 is a sensitive and diagnostically useful immunohistochemical marker of normal endometrial stroma and of endometrial stromal neoplasms. Histopathology 2001;39:273-8.

16. Ronnett BM, Zaino RJ, Ellenson LH, Kurman RJ. Endometrial adenocarcinoma. In: Kurman RJ, editor. Blaustein's pathology of the female genital tract. New York: Springer-Verlag; 2002. p. 501-59.

17. Bergholt T, Eriksen L, Berendt N, Jacobsen M, Hertz JB. Prevalence and risk factors of adenomyosis at hysterectomy. Hum Reprod 2001;16:2418-21.

18. Raju GC, Naraynsingh V, Woo J, Jankey N. Adenomyosis uteri: a study of 416 cases. Aust N Z J Obstet Gynaecol 1988;28:72-3.

19. Vercellini P, Parazzini F, Oldani S, Panazza S, Bramante T, Crosignani PG. Adenomyosis at hysterectomy: a study on frequency distribution and patient characteristics. Hum Reprod 1995;10:1160-2.

20. Seidman JD, Kjerulff KH. Pathologic findings from the Maryland Women's Health Study: practice patterns in the diagnosis of adenomyosis. Int J Gynecol Pathol 1996;15:217-21.

21. Parazzini F, Vercellini P, Panazza S, Chatenoud L, Oldani S, Crosignani PG. Risk factors for adenomyosis. Hum Reprod 1997;12:1275-9.

22. Mittal KR, Barwick KW. Endometrial adenocarcinoma involving adenomyosis without true myometrial invasion is characterized by frequent preceding estrogen therapy, low histologic grades, and excellent prognosis. Gynecol Oncol 1993;49:197-201.

23. Ritz J, Pesando JM, Notis-McConarty J, Lazarus H, Schlossman SF. A monoclonal antibody to human acute lymphoblastic leukaemia antigen. Nature 1980;283:583-5.

24. Metzgar RS, Borowitz MJ, Jones NH, Dowell BL. Distribution of common acute lymphoblastic leukemia antigen in nonhematopoietic tissues. J Exp Med 1981;154:1249-54.

25. Trejdosiewicz LK, Malizia G, Oakes J, Losowsky MS, Janossy G. Expression of the common acute lymphoblastic leukaemia antigen (CALLA gp100) in the brush border of normal jejunum and jejunum of patients with coeliac disease. J Clin Pathol 1985;38:1002-6.

26. Gusterson BA, Monaghan P, Mahendran R, Ellis J, O'Hare MJ. Identification of myoepithelial cells in human and rat breasts by ancommon acute lymphoblastic leukemia antigen antibody A12. J Natl Cancer Inst 1986;77:343-9.

27. Sato Y, Itoh F, Hinoda Y, Ohe Y, Nakagawa W, Ueda R, et al. Expression of CD10/neutral endopeptidase in normal and malignant tissues of the human stomach and colon. J Gastroenterol 1996;31:12-7.

28. Hirsch MS, Huettner PC, Cviko A, Quade BJ, Nucci MR. CD10 (CALLA) positive/h-caldesmon negative immunophenotype effectively distinguishes endometrial stromal tumors from uterine smooth muscle tumors [abstract]. Mod Pathol 2002; 15:198A. 